\title{
Penser dans la crise de l'éducation : Hannah Arendt et le problème de la contingence
}

Par ANTOINE JANVIER

Université de Liège

\section{Introduction}

On considère souvent que l'enjeu de l'éducation à la modernité est de tenir à la fois les principes d'égalité et de liberté propres aux démocraties modernes comme fin de l'éducation, et la nécessaire hiérarchie constitutive du rapport pédagogique entre le maître et l'élève, l'adulte et l'enfant, le vieux et le jeune; on considère tout aussi souvent, en conséquence, la crise de l'éducation depuis l'impossibilité de tenir ces deux exigences contradictoires, les invocations en pure perte de la seconde (vide de l'autorité, de la contrainte, de la hiérarchie) ne témoignant que de la victoire unilatérale et autodestructrice de la première ${ }^{1}$. À considérer la liberté comme liberté individuelle, les sociétés modernes ont rendu intenable le respect de contraintes collectives nécessaires à tout rapport pédagogique; à soutenir l'égalité comme égalité des individus pris abstraitement, c'est-à-dire indifféremment, quels qu'ils soient, les sociétés modernes ont conduit à l'échec le respect du rapport inégalitaire distinguant celui qui sait de celui qui ne sait pas encore.

Le célèbre article d'Hannah Arendt « La Crise de l'éducation », publié dans Partisan Review en 1958 et repris dans le recueil Beetween Past and

\footnotetext{
${ }^{1}$ Voir par exemple Alain Renaut, La Libération des enfants : Contribution à une histoire philosophique de l'enfance, Paris, Bayard/Calmann Lévy, 2002 et MarieClaude Blais, Marcel Gauchet et Dominique Ottavi, Pour une philosophie politique de l'éducation: Six questions d'aujourd'hui, Paris, Bayard, 2002.
} 
Future en $1961^{1}$, parait à bien des égards constituer l'envoi de ce vaste débat. Arendt y condamne fermement «cet assemblage de théories modernes de l'éducation, qui viennent du centre de l'Europe et consistent en un étonnant salmigondis de choses sensées et d'absurdités », ayant révolutionné « de fond en comble tout le système d'éducation, sous la bannière du progrès de l'éducation ${ }^{2}$. La destruction des méthodes traditionnelles s'est en particulier radicalisée en Amérique, en raison du «caractère politique de ce pays, qui, de lui-même, se bat pour égaliser ou effacer [to equalize or to erase], autant que possible, la différence entre jeunes et vieux, doués et non doués, c'est-à-dire finalement entre enfants et adultes et en particulier entre professeurs et élèves $»^{3}$. Mais derrière cette bruyante condamnation, dont les échos s'entendent aujourd'hui encore, Arendt développe une position originale du problème de l'éducation et de sa crise à la modernité : en réalité, Arendt lance moins ce débat qu'elle ne le traverse pour en sortir ou, plus exactement, pour le déplacer et en reformuler les termes.

\section{Penser dans l'intervalle du temps}

Il faut d'abord rappeler le statut de l'exercice auquel Arendt se livre dans ce texte. C'est en effet le terme du sous-titre du recueil, Six Exercises in Political Thought. La préface en déplie la signification : il s'agit d'exercices d'un genre qui « est différent de processus mentaux comme la déduction, l'induction et le fait de tirer des conclusions »; "leur seul but est d'acquérir de l'expérience en : comment penser $\gg{ }^{4}$.

C'est que penser ne va pas de soi. Dans sa préface, Arendt remarque que «l'appel à la pensée se fait entendre dans l'étrange entre-deux qui s'insère parfois dans le temps historique [the odd in-between period which sometimes inserts itself into historical time] où non seulement les historiens mais les acteurs et les témoins, les vivants eux-mêmes, prennent conscience d'un intervalle dans le temps qui est entièrement déterminé par des choses

${ }^{1}$ Cet ouvrage sera traduit en français en 1972 chez Gallimard, sous la direction de Patrick Lévy, avec comme titre La Crise de la culture, qui est d'abord le titre de l'un des articles de ce recueil.

${ }^{2}$ Hannah Arendt, « La Crise de l'éducation », dans Id., La Crise de la culture, trad. fr. P. Lévy (dir.), Paris, Éditions Gallimard, 1972, p. 229.

${ }^{3}$ Ibid., p. 232.

${ }^{4}$ Ibid., p. 25. 
qui ne sont plus et des choses qui ne sont pas encore $»^{1}$. Un tel entre-deux, pour Arendt, est précisément ce qui s'impose au sortir de la Seconde Guerre mondiale, comme il s'était déjà imposé à l'issue de la Première. Aussi s'efforce-t-elle d'en saisir la teneur en convoquant une parabole de Kafka, rédigée par Kafka dans les années 1920, parmi d'autres notes :

Il y a deux antagonistes : le premier le pousse de derrière, depuis l'origine. Le second barre la route devant lui. Il se bat avec les deux. Certes, le premier le soutient dans son combat contre le second car il veut le pousser en avant et de même le second le pousse en arrière. Mais il n'en est ainsi que théoriquement. Car il n'y a pas seulement les deux antagonistes en présence mais aussi, encore lui-même, et qui connaît réellement ses intentions ? Son rêve, cependant, est qu'une fois, dans un moment d'inadvertance - et il y faudrait assurément une nuit plus sombre qu'il n'y en eut jamais - il quitte d'un saut la ligne de combat et soit élevé, à cause de son expérience du combat, à la position d'arbitre sur ses antagonistes dans leur combat l'un contre l'autre.

Pour Arendt, ce que nous dit cette parabole, c'est que

du point de vue de l'homme, qui vit toujours dans l'intervalle entre le passé et le futur, le temps n'est pas un continuum, un flux ininterrompu; il est brisé au milieu, au pont où « il » se tient; et «son» lieu n'est pas le présent tel que nous le comprenons habituellement mais plutôt une brèche dans le temps que « son» constant combat, «sa » résistance au passé et au futur fait exister ${ }^{2}$.

Sans la présence de l'homme entre les forces du passé et du futur, celles-ci se seraient neutralisées ou détruites l'une l'autre depuis longtemps : l'existence humaine n'est rien d'autre que l'introduction d'une brèche dans le temps, l'insertion d'une résistance au flux du temps. Mais la parabole de Kafka semble également nous présenter la tentative des hommes pour dénier cet écart qu'ils sont: dénégation dans un rêve, le rêve de s'extirper hors du temps, et d'atteindre " une région qui surplomberait la ligne de combat»; c'est là le rêve de la métaphysique occidentale, "de Parménide à Hegel », écrit Arendt. L'effort de la pensée est-il pour autant réductible à cette dénégation dans le rêve?

Pas nécessairement. Il est possible de tenir bon dans la brèche ouverte par l'existence même. Pour comprendre, métaphoriquement, ce que peut être un tel effort de pensée, Arendt corrige l'image de Kafka : dans la mesure où deux forces différentes s'exercent sur un même point d'application, il faut se

${ }^{1}$ Ibid., p. 19.

${ }^{2}$ Ibid., p. 21. 
représenter la situation non pas dans les termes de deux forces contraires qui se font face l'une l'autre sur une même ligne, se rencontrant de front, mais dans les termes de ce que les physiciens appellent un "parallélogramme des forces ", c'est-à-dire comme une rencontre de biais entre deux forces différentes, de telle sorte que le résultat n'est pas l'immobilisation de celui qui se trouve pris entre ces forces, mais son déplacement selon une troisième force, qui résulte de l'application des deux autres. Voilà ce que serait la pensée : non pas le rêve de sortir et de juger, du dehors, un présent réduit à l'impuissance immobile qui se tient entre le passé et le futur; mais le tracé d'une tangente, ou d'une oblique - Deleuze dirait d'une ligne de fuite dans le champ de bataille dessiné par les forces du passé et du futur. On comprend alors pourquoi Arendt nous disait que ce lieu où l'homme tient bon, c'est-à-dire ne rêve ni ne se résigne, mais s'efforce de penser, c'est un lieu qui n'est pas le présent. Le présent se dédouble en cet instant-limite compris entre le passé et le futur, et en un présent-éternel qui embrasse le passé et le futur (la résignation ou le rêve). Mais ce lieu où l'homme pense et tient bon, ce «chemin frayé par la pensée», c'est un «petit tracé de nontemps que l'activité de la pensée inscrit à l'intérieur de l'espace-temps des mortels », un "petit non-espace-temps au cœur même du temps », qui «ne peut être transmis ou hérité », mais doit être découvert et frayé « laborieusement à nouveau » par chacun ${ }^{1}$.

Cet effort ne peut donc s'appuyer sur aucun système d'évidences dispensées par le sens commun ou dévoilées pour l'éternité par une philosophia perennis. C'est même à la faveur d'une mise en crise d'un tel système que la brèche entre le passé et le futur "devint une réalité tangible et un problème pour tous $\gg{ }^{2}$. C'est ainsi que l'article d'Arendt sur la crise de l'éducation rappelle l'impossibilité de penser dans les coordonnées léguées par la tradition. Une crise se caractérise par l'impuissance de «la saine raison humaine » qui « achoppe et ne permet plus de fournir de réponses » ${ }^{3}$. Elle se signale par un effondrement du sens commun :

La disparition [du] sens commun aujourd'hui est le signe le plus sûr de la crise actuelle. À chaque crise, c'est un pan du monde, quelque chose de commun à tous qui s'écroule. Comme une baguette magique la faillite du sens commun indique où s'est produit un tel effondrement ${ }^{4}$.

\footnotetext{
${ }^{1}$ Ibid., p. 24.

2 Ibid., p. 25.

${ }^{3}$ Ibid., p. 229.

${ }^{4}$ Ibid., p. 230.
} 
Mais la crise n'est pas strictement négative. Une crise «fait tomber les masques et les préjugés », ce qui « signifie tout simplement que nous avons perdu les réponses sur lesquelles nous nous appuyons généralement, sans même nous rendre compte qu'elles étaient à l'origine réponses à des questions ${ }^{1}$. La crise de l'éducation n'est donc pas le résultat de la destruction, par de saugrenus idéaux, de bonnes vieilles méthodes fondées sur de bonnes vieilles conceptions, auxquelles le bon sens imposerait de revenir. Elle procède d'une réouverture des questions par-delà les réponses qui les ont recouvertes; elle rouvre l'éducation comme problème, et donc matière à penser.

\section{La crise de l'éducation : du problème de l'égalité au problème du nouveau}

Lu sous cet angle, le texte d'Arendt prend une autre couleur. Il est vrai qu'à bien des égards, Arendt multiplie les poncifs : contre des clichés, d'autres clichés. Les méthodes pédagogiques nouvelles se fonderaient sur l'idée d'un monde propre de l'enfance à respecter. Elles soutiendraient que la capacité pédagogique du professeur est primordiale, voire suffisante, plutôt que sa maîtrise d'un sujet déterminé. Elles réclameraient un apprentissage qui procède par le jeu, «seule forme d'activité qui jaillisse spontanément de son existence d'enfant $\|^{2}$ et corresponde par conséquent à son mode d'activité « caractéristique ». Or « le conservatisme, rappelle Arendt, pris au sens de conservation, est l'essence même de l'éducation $»^{3}$. Aux évidences des « expériences modernes d'éducation [qui] ont pris des allures tout à fait révolutionnaires ${ }^{4}$, on objectera donc d'autres évidences renouant avec « la saine raison humaine » et « les règles du bon sens $»^{5}$. L'éducation n'a pas à respecter l'autonomie d'un groupe d'enfants: car elle conduirait à une tyrannie du groupe sur chaque enfant, en refusant d'assumer son rôle propre, qui est de faire grandir les enfants et donc de les amener à l'état d'adulte. On n'apprend jamais une pure forme ; et l'on n'apprend qu'à supposer que celui qui vous l'apprend sait ce qu'il vous apprend: il faudrait donc que les professeurs apprennent eux-mêmes quelque chose, leur maîtrise du savoir

\footnotetext{
${ }^{1}$ Ibid., p. 224-225.

${ }^{2}$ Ibid., p. 235.

${ }^{3}$ Ibid., p. 246.

${ }^{4}$ Ibid., p. 245-246.

${ }^{5}$ Ibid., p. 229.
} 
enseigné constituant la condition de leur autorité ${ }^{1}$. Et si l'on considère que, minimalement, l'éducation vise non à rester en enfance mais à en sortir, on conviendra que, quelle que soit la forme concrète d'apprentissage promulguée, l'argument d'une forme conforme à la condition infantile ne peut tenir : il est donc douteux qu'au motif de son adéquation à l'initiative propre de l'enfant le jeu soit un instrument pédagogique recommandable.

À ces clichés réactifs correspondent des mesures pratiques. Et Arendt ne se gêne pas pour les présenter sous une plume qui s'avère, à y prêter attention, légèrement ironique :

En Amérique, la crise actuelle résulte [...] de l'effort désespéré qui est tenté pour réformer tout le système d'éducation, c'est-à-dire pour le transformer complètement. Ce faisant, exception faite des projets qui visent à augmenter considérablement les moyens d'enseignement mis à la disposition des sciences physiques et de la technologie, on ne tente rien d'autre qu'une restauration : on rétablira une fois de plus l'autorité dans l'enseignement ; on ne jouera plus pendant les heures de classe et on fera de nouveau du travail sérieux ; on mettra l'accent non plus sur les activités extra-scolaires, mais sur les matières du programme. Enfin, on parle même de modifier les programmes actuels de formation des professeurs qui devront eux-mêmes apprendre quelque chose avant d'être lâchés auprès des enfants ${ }^{2}$.

C'est qu'en réalité, de telles mesures, Arendt les juge " catastrophiques » (these ruinous measures) $^{3}$. Elles ne voient la crise de l'éducation qu'en surface : comme une mise en crise de la hiérarchie éducative sous l'effet d'une idéologie égalitaire. Quid alors de ce combat de l'Amérique "pour égaliser ou effacer, autant que possible, la différence entre jeunes et vieux, doués et non doués, c'est-à-dire finalement entre enfants et adultes et en particulier entre professeurs et élèves »? Mais «ces facteurs généraux ne peuvent ni expliquer la crise dans laquelle nous nous trouvons actuellement,

${ }^{1}$ Ibid., p. 237.

${ }^{2}$ Idem.

${ }^{3}$ Il faut se poser la question de savoir à quelles mesures Arendt renvoie exactement par cette expression. Il semble dans un premier temps qu'elle identifie par là les mesures pédagogiques de réforme du système éducatif américain fondées sur les théories pédagogiques modernes ; mais à bien lire le mouvement du paragraphe II, qui cherche à restituer la dialectique - sans relève - entre modernisation pédagogique et restauration ( On ne tente rien d'autre qu'une restauration ») productrice de la crise, il apparaît que les mesures en question sont plutôt celles qui sont prévues dans «les réformes envisagées» (ibid., p. 237), ou, du moins, l'ensemble des mesures réformistes, qu'elle soient « progressistes » ou « restauratrices ». 
ni justifier les mesures par lesquelles on a précipité la crise ${ }^{1}$. Simplement, l'idéologie politique égalitaire rend la crise particulièrement $\left\langle\right.$ aiguë ${ }^{2}$ : elle n'en constitue pas la cause, ni le fondement. Il faut donc regarder avec méfiance ce qu'Arendt appelle un «bavardage à la mode sur un nouveau conservatisme $»^{3}$ qu'elle traverse pour son compte dans le texte dont nous parlons - bien souvent sous la forme du discours indirect libre. Un tel bavardage masque le problème plus profond : c'est l'idée même d'éducation, qu'elle soit « progressiste » ou « réactionnaire », qui est mise en cause, et pas telle ou telle de ses formes.

Que s'agit-il alors de penser dans l'éducation? Qu'est-ce qui est en crise au juste ? L'éducation pose avant tout la question du nouveau - avant celle de l'égalité et de l'inégalité, avant celle de la liberté et de la contrainte :

L'éducation est l'une des activités les plus élémentaires et les plus nécessaires de la société humaine, laquelle ne saurait jamais rester telle qu'elle est, mais se renouvelle sans cesse par la naissance, par l'arrivée de nouveaux venus ${ }^{4}$.

Pour les progressistes comme pour les réactionnaires, le problème de l'éducation touche à la question de l'individu humain. C'est un problème de bonne constitution de l'homme comme être déficient, inachevé, devant par conséquent être éduqué - ayant besoin d'un maître, selon la formule kantienne , que ce maître soit un adulte bienveillant respectant l'autonomie déjà là de l'enfant, permettant l'exercice de sa spontanéité, s'émerveillant de ses facultés pratiques, ou qu'il soit au contraire le représentant sévère de l'autorité générationnelle permettant à l'enfant d'accéder à la socialisation et aux savoirs sans lesquels il ne pourrait être ce qu'il a à être. Mais, objecte Arendt, "si l'enfant n'était pas un nouveau venu dans ce monde des hommes, mais seulement une créature vivante pas encore achevée, l'éducation ne serait qu'une des fonctions de la vie et n'aurait pas d'autre but que d'assurer la subsistance et d'apprendre à se débrouiller dans la vie, ce que tous les animaux font pour leurs petits ${ }^{5}$. Pour Arendt, la question de l'éducation est donc une question politique, au sens où ses enjeux ne concernent

\footnotetext{
${ }^{1}$ Ibid., p. 232.

${ }^{2}$ Idem.

${ }^{3}$ Ibid., p. 247.

${ }^{4}$ Ibid., p. 238.

${ }^{5}$ Idem.
} 
pas une hypothétique « nature humaine », mais une condition ${ }^{1}$ constitutivement définie par son existence collective, c'est-à-dire par le fait de la pluralité humaine à laquelle l'arrivée incessante de nouveaux venus donne toute sa puissance ${ }^{2}$. Et dans la mesure où, pour Arendt, c'est la consistance d'un «monde» qui donne à la pluralité humaine les conditions et les modalités de son inscription dans la durée, le problème de l'éducation d'un nouveau venu se concentre dans le problème du renouvellement du monde ${ }^{3}$.

\section{Introduire les nouveaux venus dans le monde}

Dans La Condition de l'homme moderne, publié en même temps que «La Crise de l'éducation » en 1958, Arendt définit le monde comme l'habitat construit par l'homme sur cette terre. On sait qu'Arendt distingue trois types d'activités humaines, trois formes de la vita activa: le travail, l'action et l'œuvre. Le travail s'inscrit dans le processus biologique du corps humain. L'action met directement les hommes en rapport entre eux — il s'agit en réalité de l'action et de la parole publiques comme manières d'assumer et de donner forme à la pluralité caractéristique de la condition humaine sur une scène publique, c'est-à-dire la politique. L'œuvre, enfin, est l'activité par laquelle l'homme institue entre lui et le milieu naturel un monde humain, c'est-à-dire un monde d'objets durables inscrivant dans l'environnement de notre existence la marque de l'humanité par-delà la précarité des individus. Ce qui caractérise l'œuvre, c'est la permanence : les «objets artificiels" (d'une table à une œuvre d'art, d'un objet simplement fabriqué à une création artistique) ne s'épuisent pas dans leur usage - contrairement aux objets de consommation destinés à la production et la reproduction de notre existence - pas plus que l'activité qui les constitue ne s'achève en et par elle-même - contrairement à la praxis politique des hommes libres sur la scène

${ }^{1}$ Sur la différence entre «nature humaine » et « condition », voir Hannah Arendt, Condition de l'homme moderne, chapitre IV « L'CEuvre», trad. fr. G. Fradier, Paris, Éditions Calmann-Lévy, 1961, rééd. Pockett, 1994, p. 41-46.

${ }^{2}$ Sur les enjeux politiques de la question de la natalité chez Arendt, voir Miguel Abensour, Hannah Arendt contre la philosophie politique?, Paris, Éditions Sens et Tonka, 2006, p. 115-150.

${ }^{3}$ Dans son article sur « Hannah Arendt, l'éducation et la question du monde » (dans Le Télémaque, $\left.\mathrm{n}^{\circ} 19,2001\right), \mathrm{p}$. 79-101, Philippe Foray a bien vu que ce qui est en jeu dans l'éducation, selon Arendt, c'est le «monde ». Il reste néanmoins en-deçà des conséquences qu'on peut en tirer et des problèmes que pose, sans les résoudre, ce déplacement arendtien des débats sur la crise de l'éducation. 
publique. En ce sens, l'œuvre institue un monde, c'est-à-dire une permanence de l'humanité par-delà la fragilité de son existence et de ses actions.

On peut définir le monde par ses deux dimensions : la place possédée par chacun, c'est-à-dire la parcelle du monde qui est la nôtre et dans laquelle nous pouvons nous retrancher du regard et du commentaire des autres, régulièrement pensée par Arendt avec la catégorie grecque de l'oïkos; l'espace inter homines, c'est-à-dire l'espace d'articulation des différentes places dans le monde et de rencontre entre les hommes, qu'Arendt appelle common world, "monde commun». L'une de ces dimensions ne peut être pensée sans l'autre : c'est dans leur mise à distance même par l'institution d'un espace commun, que la place de chacun est garantie et reconnue ; inversement, le monde commun n'est rien d'autre que l'institution de la distance et de l'articulation des places de chacun, via l'espace public comme «topos des topoï ». On trouve donc chez Arendt, comme l'a très bien démontré Étienne Tassin, une pensée

topique des lieux articulés entre eux par l'institution symbolique d'un topos de la pluralité, topos «commun» en ce qu'il inscrit la pluralité [humaine] dans la visée d'une communauté qu'aucune origine commune ne fonde ou justifie tandis qu'elle récuse par principe toute communion finale ${ }^{1}$.

Ces deux dimensions du monde sont comprises par Arendt depuis les deux cas que constituent à ses yeux respectivement le foyer familial chez les Romains et les objets fabriqués par les hommes. D'un côté, le foyer renvoie à la propriété, qui « signifiait, ni plus ni moins, avoir sa place en un certain lieu du monde et donc appartenir à la cité politique $»^{2}$. Chez les Romains, rappelle Arendt, ce foyer doit rester caché du domaine public, parce qu'il est « le lieu de la naissance et de la mort » qui doivent rester cachées aux regards des hommes : manière d'assumer que "l'homme ne sait pas d'où il vient quand il naît ni où il va quand il meurt ${ }^{3}$. D'un autre côté, les objets d'usage et les objets d'art fabriqués par les hommes durent par-delà leur usage singulier, et se distinguent des produits voués à disparaître dans la consommation qu'en fait la vie humaine pour sa reproduction. Durables, les objets du monde ont dès lors " une relative indépendance par rapport aux hommes qui les ont produits et qui s'en servent, une "objectivité" qui les fait "s'opposer", résister, au moins quelques temps, à la voracité de leurs auteurs

${ }^{1}$ Étienne Tassin, Le Trésor perdu. Hannah Arendt et l'intelligence de l'action politique, Paris, Éditions Payot, 1999, p. 533.

${ }^{2}$ Hannah Arendt, La Condition de l'homme moderne, op. cit., p. 102.

${ }^{3}$ Ibid., p. 104. 
et usagers vivants ». Arendt en conclut qu'ils « ont pour fonction de stabiliser la vie humaine » et que les hommes, « en dépit de leur nature changeante, peuvent recouvrer leur identité dans leurs rapports avec la même chaise, la même table ${ }^{1}$. Dans le foyer familial ou dans les objets fabriqués, on trouve à chaque fois une même modalité par laquelle les hommes conjurent et règlent leur finitude. Autant dire que, pour Arendt, l'institution d'un monde relève de l'effort par lequel les hommes se ressaisissent collectivement du rapport à ce qui, du fait même de leur existence, leur échappe. Le monde est permanent, ce qui veut dire : ni éternel, ni instantané, mais durable. S'il est durable, le monde requiert par conséquent une certaine continuité - une réappropriation par chaque nouveau venu, en tant que manifestation « objective » et pour ainsi dire " solide », « tangible », dit parfois Arendt, de la communauté humaine; mais il requiert également un certain renouvellement, modalité de sa réappropriation par les nouveaux venus ${ }^{2}$.

Dans ce cadre, ce que manifeste l'enfance, c'est quelque chose qui fait rupture dans ce monde: quelque chose qui dérange, ébranle et rend par conséquent possible, du même coup, le renouvellement nécessaire de ce monde. Éduquer, c'est assumer la responsabilité de la continuité du monde vis-à-vis de ces êtres nouveaux, ce qui veut dire deux choses. Premièrement, éduquer signifie présenter le monde comme sien aux êtres nouveaux: se poser comme éducateur face à l'enfant c'est lui présenter le monde comme monde, c'est-à-dire comme lieu du commun dont on procède et auquel on participe ; cela revient à dire : «Voici notre monde ${ }^{3}$. Mais éduquer signifie aussi assumer les limites de ce monde comme monde, assumer qu'il est voué à être renouvelé dans un sens que l'on ignore encore, vers une figure inconnue :

Au fond, on n'éduque jamais que pour un monde déjà hors de ses gonds ou sur le point d'en sortir, car c'est là le propre de la condition humaine que le monde soit crée par des mortels afin de leur servir de demeure pour un temps limité. Parce que le monde est fait par des mortels, il s'use ; et parce que ses habitants changent continuellement, il court le risque de devenir mortel comme eux. Pour préserver le monde de la mortalité de ses créateurs et de ses habitants, il faut constamment le remettre en place. Le problème est tout

\footnotetext{
${ }^{1}$ Ibid., p. 188 .

${ }^{2}$ Sur ce point, voir le chapitre IV de La Condition de l'homme moderne, op. cit.

${ }^{3}$ Hannah Arendt, « La Crise de l'éducation », art. cit., p. 243.
} 
simplement d'éduquer de façon telle qu'une remise en place demeure effectivement possible, même si elle ne peut jamais être définitivement assurée ${ }^{1}$.

Les utopies politiques, remarque Arendt, ont toujours porté un intérêt à la question de l'éducation: non en raison de leur contenu - valorisant la liberté et l'égalité - mais par leur fait même (ce qui donne à l'analyse d'Arendt une portée dépassant le strict domaine des ambitions révolutionnaires «progressistes »). Les utopies révolutionnaires sont en effet fondées sur l'ambition d'une régénération ou d'une transformation radicale du monde: elles trouvent donc dans l'éducation le matériau idéal de cette transformation, les nouveaux eux-mêmes. Mais le paradoxe est que, reconnaissant les enfants comme la promesse de l'avenir, les utopies politiques referment du même geste cette promesse parce qu'elles en font la promesse de leur avenir. Reconnaissant la nouveauté du monde à venir dans les enfants, elles dénient la nouveauté du nouveau dont ils sont porteurs. Comme l'écrit Arendt, « on essaie de mettre en place le nouveau comme un fait accompli, c'est-à-dire comme s'il existait déjà $»^{2}$. Dès lors,

du point de vue des nouveaux, si nouvelles que puissent être les propositions du monde adulte, elles sont nécessairement plus vieilles qu'ils ne sont euxmêmes. C'est bien le propre de la condition humaine que chaque génération nouvelle grandisse à l'intérieur d'un monde déjà ancien, et par suite former une génération nouvelle pour un monde nouveau traduit en fait le désir de refuser aux nouveaux arrivants leurs chances d'innover ${ }^{3}$.

L'enjeu de l'éducation est d'assumer la responsabilité du monde — et donc de tenir sa continuité — pour que du nouveau puisse advenir, c'est-à-dire pour que les enfants puissent être porteurs de nouveauté. C'est en ce sens que l'éducation doit être conservatrice : elle conserve un monde dont elle ne peut anticiper le renouvellement, sauf à considérer le nouveau comme un fait accompli et donc à le rendre impossible. Autrement dit, reconnaître les enfants comme porteurs de nouveauté à protéger, c'est refuser de statuer sur ce nouveau, assumer ce «monde déjà vieux » qui est le sien et le présenter comme la condition même, indiscutable, dans laquelle son renouvellement pourra se faire ${ }^{4}$.

\footnotetext{
${ }^{1}$ Ibid., p. 247.

2 Ibid., p. 227.

${ }^{3}$ Ibid., p. 228.

${ }^{4}$ Ibid., p. 247.
} 
Les problèmes soulevés dans le domaine de l'éducation ne s'y cantonnent donc pas. Ils impliquent avec eux la question du monde : «L'éducation est le point où nous décidons si nous aimons suffisamment le monde pour en assumer la responsabilité [education is the point at which we decide whether we love the world enough to assume responsability for it] ${ }^{1}$. Comment faut-il entendre cette décision?

Dans La Crise de la culture, Arendt attribue cette faculté de décision au goût - suivant une rapide lecture de la partie «Esthétique» de la troisième Critique. On a vu que le monde est la manifestation de la permanence de l'homme à lui-même, à la fois par-delà et au moyen de ses actions et de la finitude de son existence. À cet égard, les choses de l'art apparaissent comme mondaines par excellence : ce qui les caractérise est leur durabilité - on ne les consomme pas, mais on n'en use pas non plus comme de simples objets fabriqués. Cependant, Arendt remarque que cette mondanité n'est pas une propriété des objets artistiques. Elle relève d'une décision, qu'Arendt identifie dans la présomption arbitraire du jugement de goût à l'accord de tous avec mon appréciation subjective :

L'activité du goût décide [the activity of taste decides] comment voir et entendre ce monde, indépendamment de l'utilité et des intérêts vitaux qu'il a pour nous, décide ce que les hommes y verront et y entendront. Le goût juge le monde en son apparition et en sa mondanité ${ }^{2}$.

Le goût décide, à propos d'une chose, de sa mondanité en tant qu'il l'estime mondaine : ce qui veut dire que la chose est dite du monde dans le même temps où la mondanité du monde est définie, c'est-à-dire dans le même temps où ce qui perdure de l'homme dans l'homme est déterminé. Le goût, par conséquent, décide du monde dans le même temps où il décide de l'homme - et c'est pourquoi il fonde l'activité politique de persuasion, excluant à la fois la coercition de la violence physique et la coercition de la vérité. Le goût ne porte sur la mondanité d'une chose, et donc sur la mondanité du monde lui-même, qu'en portant sur l'humanité de l'homme. Arendt peut alors écrire :

Ranger le goût, principale activité culturelle, parmi les facultés politiques de l'homme, a quelque chose de si étrange que je dois ajouter un autre fait beau-

${ }^{1}$ Ibid., p. 251. Je souligne l'expression dont j'ai légèrement corrigé la traduction (l'édition française traduit « où se décide »).

${ }^{2}$ Hannah Arendt, « La Crise de la culture », dans Id., La Crise de la culture, op. cit., p. 284. 
coup plus familier, mais peu envisagé théoriquement, à ces considérations. Nous savons très bien avec quelle rapidité les gens se reconnaissent, comment sans équivoque ils peuvent se sentir en communion lorsqu'ils découvrent une parenté en matière de goûts et de dégoûts. Du point de vue de cette expérience courante, tout se passe comme si le goût décidait non seulement comment voir le monde, mais aussi qui s'appartient en ce monde ${ }^{1}$.

Il faut bien lire : le goût ne décide pas qui appartient à ce monde, mais qui $s$ 'appartient en ce monde, au sens où dans le goût se joue la décision de se sentir appartenir dans un monde, de se sentir participant du monde. C'est bien en ce sens qu'Arendt semble l'entendre lorsqu'elle définit la crise comme crise du sens commun - du sensus communis, dont on sait que Kant le considérait comme révélé dans le goût. C'est bien en ce sens qu'elle rappelle que «l'éducation est le point où se décide si nous aimons assez le monde pour en assumer la responsabilité », ce qui pourrait se traduire : l'éducation est le point où se décide si nous goûtons au monde qui est le nôtre, c'est-à-dire si nous l'éprouvons comme monde — ou non.

\section{La crise de l'éducation}

On peut maintenant poser à nouveaux frais le problème de la crise de l'éducation. Sur ce point, les remarques d'Arendt sur la disparition de l'autorité en éducation sont significatives. Tout en estimant que l'autorité est constitutive du rapport éducatif puisqu'elle est la forme objective de l'assomption subjective du monde comme monde par les adultes devant les enfants, et donc tout en définissant la crise de l'éducation notamment comme perte et effondrement de l'autorité, Arendt se garde bien d'y voir là une cause, ou même de la rattacher à des illusions pédagogiques égalitaires. Elle rapporte plutôt cet effondrement à ses conditions, à savoir la perte du sentiment du monde, plus exactement au dégoût pour un monde qui n'est précisément, pour cette raison, plus vécu comme tel :

L'autorité a été abolie par les adultes et cela ne peut signifier qu'une chose : que les adultes refusent d'assumer la responsabilité du monde dans lequel ils ont placé les enfants. [...] L'homme moderne ne pouvait exprimer plus clairement son mécontentement envers le monde et son dégoût pour les choses telles qu'elles sont qu'en refusant d'en assumer la responsabilité pour ses enfants. C'est comme si, chaque jour, les parents disaient : « En ce monde

${ }^{1}$ Ibid., p. 285. 
même nous ne sommes pas en sécurité chez nous ; comment s'y mouvoir, que savoir, quel bagage acquérir sont pour nous aussi des mystères. Vous devez essayer de faire de votre mieux pour vous en tirer ; de toute façon vous n'avez pas de compte à nous demander. Nous sommes innocents, nous nous lavons les mains de notre sort $»{ }^{1}$.

Ce dont la crise de l'éducation témoigne, c'est donc au fond d'un dégoût. Il est connu qu'Arendt rattache la modernité à un vaste processus, complexe, d'aliénation par rapport au monde ${ }^{2}$, dont le totalitarisme constitue un pointlimite de cristallisation. C'est ici aux conséquences de ce processus sur le plan de la subjectivité qu'Arendt porte son attention : «En ce monde même nous ne sommes pas en sécurité chez nous ». Je laisserai donc de côté ici les conditions objectives de l'aliénation par rapport au monde, par ailleurs bien connues $^{3}$, pour conclure sur l'enjeu, dans le cadre des questions d'éducation, de cette problématisation de la subjectivité contemporaine. Je le ferai au moyen d'un bref détour par l'autobiographie intellectuelle de Stefan Zweig écrite en 1942, Le Monde d'hier (Die Welt von Gestern).

Le Monde d'hier est le récit d'une rupture, et des effets de cette rupture. Une rupture objective entre le monde d'hier, celui d'avant la guerre de 1914, celui de l'enfance et de la jeunesse de Zweig, celui de son éducation; et ce qui suit ce monde d'hier, la séquence qu'Enzo Traverso a qualifié de "guerre civile européenne", de 1914 à la fin de la Seconde Guerre mondiale ${ }^{4}$. Une rupture subjective aussi - répercussion dans la manière dont Zweig se rapporte à soi, aux autres et au monde, du changement de monde : d'abord comme une faille toute extérieure et inassimilable entre l'humanisme dont à titre d'écrivain Zweig se sent tout particulièrement porteur et le réel qui s'y soustrait; ensuite comme une faille intérieure entre ce même humanisme peu à peu assumé comme pur idéal irréalisable, en

\footnotetext{
${ }^{1}$ Ibid., p. 244-245.

${ }^{2}$ Suivant le dernier chapitre de La Condition de l'homme moderne, on peut distinguer entre une aliénation de l'homme par rapport à la Terre engagée par les grandes découvertes de la Renaissance et la conquête spatiale, une aliénation par rapport à la Nature propre à la science moderne, et une aliénation par rapport au monde provoquée par la généralisation et l'intensification du mode de production capitaliste à l'échelle européenne puis mondiale.

${ }^{3}$ Voir notamment l'ouvrage d'Étienne Tassin déjà cité, et celui de Marie-Claire Caloz-Tschopp, Les Sans-État dans la philosophie d'Hannah Arendt, Lausanne, Éditions Payot, 2000.

${ }^{4}$ Enzo Traverso, 1914-1945 : La Guerre civile européenne, Paris, Éditions Hachette, 2009.
} 
contradiction avec la réalité, et la conscience de cette même réalité et des types d'intervention qu'il convient d'y construire ; enfin, comme une béance du sujet lui-même qui s'effondre face au constat de son impuissance à vivre, doté de cette armature idéologique humaniste, dans le monde des années 1930. Ce qui est frappant, c'est qu'à l'assimilation progressive par Zweig de la rupture entre le monde d'hier et celui de l'entre-deux guerres, c'est-à-dire en réalité celui $\mathrm{du} \mathrm{XX}^{\mathrm{e}}$ siècle se constituant, répond un double paradoxe : la conscience progressive de la rupture s'accompagne d'une conscience de l'impossibilité de se départir de son humanisme, de sa foi en une réconciliation universelle des peuples à laquelle le poète, l'écrivain, l'artiste ont vocation à prendre part activement par-delà les clivages partisans de la politique, entre les classes ou entre nations; le décalage et l'inadaptation de l'idéologie humaniste de Zweig a pour effet paradoxal une lucidité plus grande que celle de ses contemporains sur les événements de cet aprèsguerre, comme si l'incapacité à voir venir la guerre de 1914 avait provoqué une claire appréhension de sa propre naïveté, devenant par conséquent le moyen même d'évaluer ou de mesurer les dangers courus par les hommes.

L'intérêt du texte de Zweig pour notre propos est de définir cette rupture, à la fois objective et subjective, qui ouvre le $\mathrm{XX}^{\mathrm{e}}$ siècle, comme la rupture entre un monde de la sécurité et un monde du bouleversement menaçant, de la fin virtuelle, de la rupture latente. Ce qui se passe avec la guerre de 1914, ce n'est pas seulement le surgissement d'un événement imprévisible dans un ordre qui avait oublié sa propre historicité : c'est l'irruption de la destruction de la possibilité même d'un ordre du monde. Significativement, le premier chapitre de l'ouvrage qui décrit l'Empire austro-hongrois de la fin du XIX siècle s'intitule «Le Monde de la sécurité », «Die Welt der Sicherheit ${ }^{1}$. Il ne faut pas donner trop de crédit à la valeur descriptive de ces pages, qu'il faut lire à la lumière rétrospective de la catastrophe de la guerre mondiale. Elles circonscrivent, par contraste, la teneur du réel qui détruit le sentiment de progression morale de l'humanité et d'avènement d'un monde pacifié et réconcilié. Réel ne veut pas dire ici réalité objective, comme si Le Monde d'hier n'était au fond qu'un travail d'adaptation entre une Weltanschauung autrefois adéquate aux structures objectives auxquelles elle était accordée, et un monde nouveau, celui de l'entre-deux-guerres, désormais inconciliable avec ce qui ne peut dès lors plus avoir d'autre statut que celui de rêve - plus encore que de souvenir. Réel doit s'entendre ici comme ce qui, de la réalité signifiante, est retranché,

${ }^{1}$ Arendt y fera d'ailleurs allusion en ouverture du deuxième volume des Origines $d u$ totalitarisme consacré à l'impérialisme. 
coupé, dans l'institution même de cette réalité, de telle sorte qu'il ne puisse structurellement y faire retour que sous la forme d'un cauchemar, d'une hallucination horrible qui inquiète radicalement la réalité en question parce qu'elle témoigne d'un «inassimilable». Dans Le Siècle, Alain Badiou a qualifié le $\mathrm{XX}^{\mathrm{e}}$ siècle comme épris d'une passion du réel, au sens lacanien du terme, par quoi il fallait entendre avant tout que les acteurs du siècle se donnent pour tâche d'affronter ce qui dérange et menace de détruire l'ordre symbolique existant, ce qui soustrait l'ordre du sens à son évidence et à sa garantie, ce qui le fragilise et l'ouvre à la double possibilité de sa destruction et de sa régénération ${ }^{1}$. C'est bien l'avènement d'une telle conscience que nous lisons dans Le Monde d'hier, sous la forme de l'impossible tentative de son auteur pour l'intégrer subjectivement: le monde est comme tel contingent.

Il me semble que c'est à un tel phénomène qu'Arendt est confrontée dans la crise de l'éducation. Ce qui caractérise les enfants, ce n'est pas tellement qu'ils sont nouveaux, mais qu'ils peuvent l'être comme ne pas l'être : qu'ils manifestent l'irruption de la contingence dont la maîtrise, c'està-dire l'inscription dans un ordre du monde comme opérateur de destruction et/ou de régénération, de reproduction et/ou de transformation, est par définition problématique, quelle que soit la forme de la société considérée ${ }^{2}$. Mais ce qui, peut-être, singularise la modernité et se trouve radicalisé par la première moitié du $\mathrm{XX}^{\mathrm{e}}$ siècle, c'est que l'éducation, comme lieu paradoxal d'une impossible maîtrise de la contingence, soit indissociable d'un risque ou d'une menace vécue comme telle par la société moderne, à savoir la menace de sa fragilité - la contingence de l'organisation sociale elle-même ${ }^{3}$. La

\footnotetext{
${ }^{1}$ Alain Badiou, Le Siècle, Paris, Éditions du Seuil, 2005.

2 Comme le dit Pierre Clastres dans la Chronique des Indiens Guayaki, «[T]oute naissance est vécue dramatiquement par le groupe en son entier, elle n'est pas simplement addition d'un individu supplémentaire à telle ou telle famille, mais une cause de déséquilibre entre le monde des hommes et l'univers des puissances invisibles, la subversion d'un ordre que le rituel doit s'attacher à rétablir » (Pierre Clastres, Chronique des Indiens Guayaki, Paris, Éditions Plon, 1972, rééd. Pockett, 2001 p. 10). On peut suivre à la trace, dans le texte de Clastres, tous les temps critiques où se joue l'équilibre de la société, et où se décide, par conséquent, sa permanence : y sont tout particulièrement important les rituels qui rythment le développement de l'existence jusqu'à l'âge adulte, recoupant les moments où cette existence devient susceptible d'en produire d'autres.

${ }^{3}$ Contingence directement ordonnée, chez Arendt, à la structuration des rapports sociaux en régime de production capitaliste comme régime d'expropriations répétées
}

\section{6}


force du texte d'Arendt, c'est d'attirer notre attention sur ceci que, quels qu'en soient les programmes, quelles que soient les finalités attribuées aux activités pédagogiques, l'éducation comme objet d'interrogation et de réflexion témoigne de la fragilité du monde. Peut-être cherche-t-elle également à nous forcer à penser l'éducation dans l'horizon d'une contingence inassimilable, en soulevant dans le même temps le problème des modes de constitution du commun et celui des formes de la permanence du monde.

et croissantes. Voir Hannah Arendt, La Condition de l'homme moderne, op. cit., p. 319-326. 